\title{
CONSTRUCTION OF MIXED AUTOMORPHIC FORMS
}

\author{
YOUNGJU CHOIE
}

(Received 19 November 1996; revised 21 March 1997)

Communicated by W. W. L. Chen

\begin{abstract}
It has been known that mixed automorphic forms arise naturally as holomorphic forms on elliptic varieties and that they include classical automorphic forms as a special case. In this paper, we show how to construct mixed automorphic forms of type $(k, l)$ from elliptic modular forms to give nontrivial examples of mixed automorphic forms.
\end{abstract}

1991 Mathematics subject classification (Amer. Math. Soc.): primary 11F55, $11 \mathrm{~F} 60$.

\section{Introduction}

The concept of mixed automorphic forms was introduced by Stiller [9] in 1984. It turns out that mixed automorphic forms arise naturally as holomorphic forms on elliptic varieties and that they include classical automorphic forms as a special case.

Given non-negative integers $k$ and $l$, mixed cusp forms of type $(k, l)$ for a discrete subgroup $\Gamma \subset S L(2, \mathscr{R})$ are defined using automorphy factors of the form $J(M, \tau)^{k} J(\chi(M), \omega(\tau))^{l}$, where $J(M, \tau)=c \tau+d$ for $M=\left(\begin{array}{ll}a & b \\ c & d\end{array}\right) \in \Gamma$ and $\omega$ is a holomorphic map of the Poincaré upper half plane satisfying $\omega(M(\tau))=\chi(M)(\omega(\tau))$ with respect to a homomorphism $\chi: \Gamma \rightarrow S L(2, \mathscr{R})$. Mixed cusp forms of type $(2, l)$ can be interpreted as holomorphic forms of the highest degree on an elliptic variety (cf. [1, 2]), and various aspects of such cusp forms were studied extensively by Lee (see for example $[3,4,6]$ ). Mixed automorphic forms of several variables have also been investigated (cf. [5, 7]).

However, unfortunately, non-trivial examples of mixed automorphic forms have never been discussed in the literature. In this paper, we give non-trivial examples of

This work was partially supported by BSRI 96-1431 and KOSEF 971-0101-003-2.

(C) 1997 Australian Mathematical Society 0263-6115/97\$A2.00+0.00 
such forms. We show how to construct mixed automorphic forms of type $(k, l)$ from elliptic modular forms. We follow definitions and notation given in [6].

\section{Mixed automorphic forms}

Let $\Gamma \subset S L(2, \mathscr{R})$ be a Fuchsian group of the first kind, and let $\chi: \Gamma \rightarrow S L(2, \mathscr{R})$ be a homomorphism of groups. Thus both $\Gamma$ and the image $\chi(\Gamma)$ of $\Gamma$ under $\chi$ operate on the Poincaré upper half plane $\mathscr{H}=\{\tau \in \mathscr{C} \mid \operatorname{Im}(\tau)>0\}$ by linear fractional transformations. Let $\omega: \mathscr{H} \rightarrow \mathscr{H}$ be a $\Gamma$-equivariant holomorphic map, that is, a holomorphic map that satisfies $\omega(M(\tau))=\chi(M) \omega(\tau)$ for all $M \in \Gamma$ and $\tau \in \mathscr{H}$. We assume that the image of a parabolic element of $\Gamma$ under $\chi$ is a parabolic element in $\chi(\Gamma)$. Given a cusp $s$ of $\Gamma$ we set

$$
\Delta_{s}=\{\sigma \in S L(2, \mathscr{R}) \mid \sigma(\infty)=s\},
$$

and let $\Delta$ be the union $\bigcup \Delta_{s}$ of $\Delta_{s}$ for all cusps $s$ of $\Gamma$. We assume that the homomorphism $\chi$ can be extended to a mapping $\chi: \Gamma \cup \Delta \rightarrow \mathscr{C}$.

Given a pair of non-negative even integers $k$ and $l$, we set $J_{\omega, X}^{k, l}(M, \tau)=(c \tau+$ $d)^{k}\left(c^{\prime} \omega(\tau)+d^{\prime}\right)^{l}$ for

$$
\left.M=\left(\begin{array}{ll}
a & b \\
c & d
\end{array}\right) \in \Gamma \cup \Delta, \quad \chi(M)=\left(\begin{array}{ll}
a^{\prime} & b^{\prime} \\
c^{\prime} & d^{\prime}
\end{array}\right) \in S L(2, \mathscr{R})\right) .
$$

Then $J_{\omega, x}^{k, l}:(\Gamma \cup \Delta) \times \mathscr{H} \rightarrow \mathscr{C}$ is a factor of automorphy, that is, it satisfies the condition

$$
J_{\omega \cdot X}^{k, l}\left(M_{1} M_{2}, \tau\right)=J_{\omega \cdot \chi}^{k, l}\left(M_{1}, M_{2}(\tau)\right) \cdot J_{\omega \cdot \chi}^{k, l}\left(M_{2}, \tau\right)
$$

for all $M_{1}, M_{2} \in \Gamma$ and $\tau \in \mathscr{H}$.

Let $s$ be a cusp of $\Gamma$, and let $\sigma$ be an element of $S L(2, \mathscr{R})$ such that $\sigma(\infty)=s$. We set $\left(\left.f\right|_{k, l} \sigma\right)(\tau)=J_{\omega, \chi}^{k, l}(\sigma, \tau)^{-1} f(\sigma \tau)$ and we have the Fourier expansion of $\left(\left.f\right|_{k, l} \sigma\right)$ at $\infty$ of the form

$$
\left(\left.f\right|_{k, l} \sigma\right)(\tau)=\sum_{n \geq n_{0}} a_{n} e^{2 \pi i n \tau / h},
$$

which is called the Fourier expansion of $f$ at $s$.

DEFINITION 2.1. Let $\Gamma, \omega$, and $\chi$ be as above. A mixed automorphic form of type $(k, l)$ associated to $\Gamma, \omega$ and $\chi$ is a holomorphic function $f: \mathscr{H} \rightarrow \mathscr{C}$ satisfying the following conditions:

(i) $f(M \tau)=J_{\omega, x}^{k, l}(M, \tau) f(\tau)$ for all $M \in \Gamma$. 
(ii) The Fourier coefficients $a_{n}$ of $f$ at each cusp $s$ satisfy the condition that $n \geq 0$ whenever $a_{n} \neq 0$.

The holomorphic function $f$ is a mixed cusp form if (ii) is replaced with the following condition:

(ii) $)^{\prime}$ The Fourier coefficients $a_{n}$ of $f$ at each cusp $s$ satisfy the condition that $n>0$ whenever $a_{n} \neq 0$.

We shall denote by $S_{k . l}(\Gamma, \omega, \chi)$ the space of mixed cusp forms of type $(k, l)$ associated to $\Gamma, \omega$ and $\chi$.

REMARK 2.2. If $S_{m}(\Gamma)$ denotes the space of cusp forms of weight $m$ for $\Gamma$, then we have

$$
S_{k .0}(\Gamma, \omega, \chi)=S_{k}(\Gamma), \quad S_{k, l}(\Gamma, \mathrm{id}, \mathrm{id})=S_{k+l}(\Gamma) .
$$

On the other hand for $k=0$ the elements of $S_{0 . l}(\Gamma, \omega, \chi)$ are generalized automorphic forms of weight $l$ in the sense of Hoyt and Stiller (see for example, [9, p. 31]).

Despite the importance of the theory of mixed cusp forms, the only known examples in the literature are those in the above Remark 2.2. The next section is concerned with the construction of other examples.

\section{Constructions}

In this section we describe how to construct mixed cusp forms from elliptic modular forms. This will generate non-trivial examples of mixed automorphic forms.

THEOREM 3.1. Let $\omega(\tau)=(\alpha \tau+\beta) /(\gamma \tau+\delta)$, where $w=\left(\begin{array}{ll}\alpha & \beta \\ \gamma & \delta\end{array}\right) \in S L(2, \mathscr{R})$. Then, for any $M \in \Gamma$, note that $\chi(M)=w M w^{-1}$. Let $f_{k+l}$ be in $S_{k+l}(\Gamma)$. Then

$$
g(\tau)=(\gamma \tau+\delta)^{l} f_{k+l}(\tau)
$$

is in $S_{k . l}(\Gamma, \omega, \chi)$.

PROOF. Since $\omega(M \tau)=\chi(M)(\omega(\tau))$, for any $M \in S L(2, \mathscr{R})$, we get $\chi(M)=$ $\omega M \omega^{-1}$, with $\omega=\left(\begin{array}{ll}\alpha & \beta \\ \gamma & \delta\end{array}\right) \in S L(2, \mathscr{R})$.

Now, first of all, the function $g(\tau)$ is a well-defined holomorphic map from $\mathscr{H}$ to $\mathscr{C}$. By simple computation, it is easy to check the functional equation of $g$ : for any $M \in \Gamma$,

$$
g(M(\tau))=J_{\omega . \chi}^{k . l}(M, \tau) g(\tau), \quad \text { for } \quad \chi(M)=w M w^{-1} .
$$


Next, we need to check the holomorphic condition of $g$ at each cusp of $\Gamma$; let $s$ be any cusp in $\Gamma$. Take $\sigma \in S L(2, \mathscr{R})$ such that $\sigma(\infty)=s$. Then, $\sigma^{-1} \Gamma_{s} \sigma=$ $\left\langle\left(\begin{array}{ll}1 & h \\ 0 & 1\end{array}\right)\right|$ some $\left.h \in \mathscr{Z}\right\rangle$ with $\Gamma_{s}=\{M \in \Gamma \mid M(s)=s\}$. Hence, it can be checked that $\left(\left.g\right|_{k, l} \sigma\right)(\tau+h)=\left(\left.g\right|_{k . l} \sigma\right)(\tau)$. This implies that there exists a function $H(z)$ on $\mathscr{K} \backslash\{0\}$ such that

$$
\left(\left.g\right|_{k . l} \sigma\right)(\tau)=H\left(e^{2 \pi i \tau / h}\right), \quad \tau \in \mathscr{H},
$$

where $\mathscr{K}$ is the unit disk (see, for instance, [8, p. 39]). On the other hand, since $f_{k+1}$ is a holomorphic elliptic modular form on $\Gamma$, at each cusp $s$ on $\Gamma$ it has a Fourier expansion of the form

$$
\left(c_{\sigma} \tau+d_{\sigma}\right)^{-k-l} f_{k+1}(\sigma(\tau))=\sum_{n>0}^{\infty} a(n) e^{2 \pi i n \tau / h}, \quad \sigma=\left(\begin{array}{cc}
* & * \\
c_{\sigma} & d_{\sigma}
\end{array}\right) .
$$

Thus, we have

$$
\begin{aligned}
\left(\left.g\right|_{k, l} \sigma\right)(\tau) & =(\gamma \tau+\delta)^{l}\left(c_{\sigma} \tau+d_{\sigma}\right)^{-k-l} f_{k+l}(\sigma(\tau)) \\
& =(\gamma \tau+\delta)^{\prime} \sum_{n>0}^{\infty} a(n) e^{2 \pi i n \tau / h} .
\end{aligned}
$$

Since $v^{l} e^{-2 \pi v n} \rightarrow 0$ as $n \rightarrow \infty$, with any $v=\operatorname{Im}(\tau)$, the above expansion guarantees the holomorphic condition of $g$ at the cusp $s$. Hence, we see that $g \in S_{k, l}(\Gamma, \omega, \chi)$. This proves the theorem.

We now give a more general theorem on the construction of mixed automorphic forms.

THEOREM 3.2. Let $\omega: \mathscr{H} \rightarrow \mathscr{H}$ be any $\Gamma$-equivariant holomorphic map, that is, $\omega(M(\tau))=\chi(M)(\omega(\tau))$ for every $M \in \Gamma$. Furthermore, we impose the condition on $\chi$ such that the image of a translation of the form $\tau \rightarrow \tau+h$ under $\chi$ is a translation $\omega \rightarrow \omega+h_{\chi}$ for some $h_{\chi} \in \mathscr{R}$. Let us take an element $N=\left(\begin{array}{ll}\alpha & \beta \\ \gamma & \delta\end{array}\right) \in S L(2, \mathscr{R})$. Then, for any elliptic cusp form $f_{l} \in S_{l}\left(N \chi(\Gamma) N^{-1}\right)$ and elliptic modular form $g_{k}$ of weight $k$ in $\Gamma$,

$$
h_{N}(\tau)=(\gamma \omega(\tau)+\delta)^{-l} f_{l}(N \omega(\tau)) g_{k}(\tau)
$$

is an element of $S_{k . l}(\Gamma, \omega, \chi)$.

ProOF. Let $\chi(M)=\left(\begin{array}{ll}a^{\prime} & b^{\prime} \\ c^{\prime} & d^{\prime}\end{array}\right)$, for any $M=\left(\begin{array}{ll}a & b \\ c & d\end{array}\right) \in \Gamma$. Then we have

$$
\begin{aligned}
h_{N}(M \tau) & =(\gamma \omega(M \tau)+\delta)^{-l} f_{l}(N \omega(M \tau)) g_{k}(M \tau) \\
& =(c \tau+d)^{k}\left(c^{\prime} \omega(\tau)+d^{\prime}\right)^{\prime}(\gamma \omega(\tau)+\delta)^{-l} f_{f}(N \omega(\tau)) g_{k}(\tau)
\end{aligned}
$$


since $\omega(M \tau)=\chi(M)(\omega(\tau)), f_{l} \in S_{l}\left(N \chi(\Gamma) N^{-1}\right)$ and $g_{k}$ is an elliptic modular form on $\Gamma$. Now we need to check the holomorphic condition of $h_{N}$ at each cusp $s$ of $\Gamma$ :

Let $s$ be a cusp of $\Gamma$. Take $\sigma=\left(\begin{array}{ll}a_{\sigma} & b_{\sigma} \\ c_{\sigma} & d_{\sigma}\end{array}\right) \in S L(2, \mathscr{R})$ satisfying $\sigma(\infty)=s$. Note that

$$
\begin{gathered}
\left.\sigma^{-1} \Gamma_{s} \sigma=\left\langle\left(\begin{array}{ll}
1 & h \\
0 & 1
\end{array}\right)\right| \text { some } h \in \mathscr{Z}\right\rangle \subseteq \sigma^{-1} \Gamma \sigma, \\
\text { with } \Gamma_{s}=\{M \in \Gamma \mid M s=s\} .
\end{gathered}
$$

Since we can see that $\left(\left.h_{N}\right|_{k, l} \sigma\right)(\tau+h)=\left(\left.h_{N}\right|_{k . l} \sigma\right)(\tau)$, there exists a function $H(z)$ on $\mathscr{K} \backslash\{0\}$ such that $\left(\left.h_{N}\right|_{k, l} \sigma\right)(\tau)=H\left(e^{2 \pi i \tau / h}\right), \tau \in \mathscr{H}$, where $\mathscr{K}$ is the unit disk. To see the holomorphic condition at each cusp $s$ in $\Gamma$, we need to consider the Fourier expansions of $f_{l}$ and $g_{k}$ at cusps:

Firstly, at each cusp $s$ in $\Gamma$, let the Fourier expansion of $g_{k}$ be $\left(\left.g_{k}\right|_{k} \sigma\right)(\tau)=$ $\left(c_{\sigma} \tau+d_{\sigma}\right)^{-k} g_{k}(\sigma(\tau))=\sum_{n \geq 0} b(n) e^{2 \pi i n \tau / h}$, for $\sigma$ as above. Next, because the image of a parabolic element in $\Gamma$ under $\chi$ is also parabolic, if $s$ is a cusp in $\Gamma$, then $\omega(s)$ is a cusp in $\chi(\Gamma)$ and $N \omega(s)$ is a cusp in $N \chi(\Gamma) N^{-1}$. Furthermore, since the image of a translation of the form $\tau \rightarrow \tau+h$ under $\chi$ is a translation $\omega \rightarrow \omega+h_{\chi}$ for some $h_{\chi} \in \mathscr{R}$, we have $\omega(\infty)=\infty$. Thus, $N \omega(\sigma(\infty))=N \omega(s)$ implies that $N \chi(\sigma)(\infty)=N \omega(s)$. This implies that

$$
\chi(\sigma)^{-1} N^{-1} \Gamma_{N \omega(s)} N \chi(\sigma) \subseteq \chi(\sigma)^{-1} \chi(\Gamma) \chi(\sigma),
$$

with $\Gamma_{N \omega(s)}=\left\{A \in N_{\chi}(\Gamma) N^{-1} \mid A(N \omega(s))=N \omega(s)\right\}$. Hence, we have that, for $\chi(\sigma)=\left(\begin{array}{ll}a_{\sigma}^{\prime} & b_{\sigma}^{\prime} \\ c_{\sigma}^{\prime} & d_{\sigma}^{\prime}\end{array}\right)$

$$
\left(\left(a_{\sigma}^{\prime} \gamma+c_{\sigma}^{\prime} \delta\right) \omega(\tau)+\left(b_{\sigma}^{\prime} \gamma+d_{\sigma}^{\prime} \delta\right)\right)^{-l} f_{l}\left(N_{\chi}(\sigma) \omega(\tau)\right)=\sum_{n>0} a(n) e^{2 \pi i \omega(\tau) n / h_{\chi}},
$$

from the cusp condition of $f_{l}$. This shows that

$$
\begin{aligned}
\left(\left.h_{N}\right|_{k, l} \sigma\right) & (\tau) \\
\quad= & \left(\left(a_{\sigma}^{\prime} \gamma+c_{\sigma}^{\prime} \delta\right) \omega(\tau)+\left(b_{\sigma}^{\prime} \gamma+d_{\sigma}^{\prime} \delta\right)\right)^{-l} f_{l}(N \chi(\sigma) \omega(\tau))\left(c_{\sigma} \tau+d_{\sigma}\right)^{-k} g_{k}(\sigma(\tau)) \\
\quad= & \left(\sum_{n>0} a(n) e^{2 \pi i \omega(\tau) n / h_{x}}\right)\left(\sum_{n \geq 0} b(n) e^{2 \pi i \tau n / h}\right),
\end{aligned}
$$

This shows that $h_{N}(\tau)$ is holomorphic at each cusp $s$ of $\Gamma$.

REMARK 3.3. (1) If we take $\omega$ to be any linear fractional transformation, that is, $\omega=\left(\begin{array}{ll}a & b \\ c & d\end{array}\right) \in S L(2, \mathscr{R})$, in the theorem, then $\omega(\tau)$ should be of the form 
$\omega(\tau)=\tau+b$, for the image of a translation of the form $\tau \rightarrow \tau+h, h \in \mathscr{Z}$ under $\chi$ is a translation $\omega \rightarrow \omega+h_{\chi}$ for some $h_{\chi} \in \mathscr{R}$. Thus,

$$
h_{N}(\tau)=(\gamma \tau+\delta)^{\prime} f_{l}(N \tau) g_{k}(\tau)
$$

is the mixed cusp form given in Remark 2.1

(2) Let $f_{l} \in S_{l}\left(N_{\chi}(\Gamma) N^{-1}\right), g_{k} \in S_{k}(\Gamma)$. If we take $N=$ id in Theorem 3.2, $h_{N}(\tau)=f_{l}(\omega(\tau)) g_{k}(\tau)$ is a mixed automorphic form of type $(k, l)$ associated with $\Gamma, \omega, \chi$.

(3) Let $f_{l} \in S_{l}(\Gamma), g_{k} \in S_{k}(\Gamma)$. If we take $N=\left(\begin{array}{cc}0 & -1 \\ 1 & 0\end{array}\right)$, then

$$
h_{N}(\tau)=\omega(\tau)^{-l} f_{l}\left(\frac{-1}{\omega(\tau)}\right) g_{k}(\tau)
$$

is a mixed automorphic form of type $(k, l)$ associated with $\Gamma, \omega, \chi$.

\section{References}

[1] B. Hunt and W. Meyer, 'Mixed automorphic forms and invariants of elliptic surfaces', Math. Ann. 271 (1985), 53-80.

[2] M. H. Lee, 'Mixed cusp forms and holomorphic forms on elliptic varieties', Pacific J. Math. 132 (1988), 363-370.

[3] — - 'Periods of mixed cusp forms', Manuscripta Math. 73 (1991), 163-177.

[4] —_. 'Mixed cusp forms and Poincaré series', Rocky Mountain J. Math. 23 (1993), 1009-1022.

[5] _ , 'Mixed Siegel modular forms and Kuga fiber varieties', Illinois J. Math. 38 (1994), 692700.

[6] _ - 'Rational structures on mixed cusp forms', Panamer. Math. J. 4 (1994), 81-100.

[7] —-, 'Mixed automorphic vector bundles on Shimura varieties', Pacific J. Math. 173 (1996), $105-126$.

[8] T. Miyake, Modular forms (Springer, Heidelberg, 1989).

[9] P. Stiller, Special values of Dirichlet series, monodromy, and the periods of automorphic forms, Mem. Amer. Math. Soc., 299 (Amer. Math. Soc., Providence, 1984).

Department of Mathematics

Pohang University of Science and Technology

Pohang

Korea 790-784

e-mail: yjc@vision.postech.ac.kr 